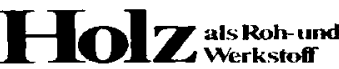

\section{Sorptionsverhalten von UF-Spanplatten vor und nach einer Trocknung bei $103^{\circ} \mathrm{C}$}

G. Böhner, Institut für Holzforschung d. Univ. München, Winzerer Straße 45, 8000 München 40, und

E. Roffael, Fraunhofer-Institut für Holzforschung, Bienroder Weg 54E, 3300 Braunschweig

Subject: Sorptional behaviour of UF-bonded particleboards before and after drying at $103{ }^{\circ} \mathrm{C}$.

Material und Methode: Für drei UF-gebundene Spanplatten wurden die Sorptionsisothermen nach zwei Sequenzen ermittelt: Adsorption - Desorption - Trocknung bei $103{ }^{\circ} \mathrm{C}$ und Trocknung bei $103^{\circ} \mathrm{C}$-Adsorption - Desorption.

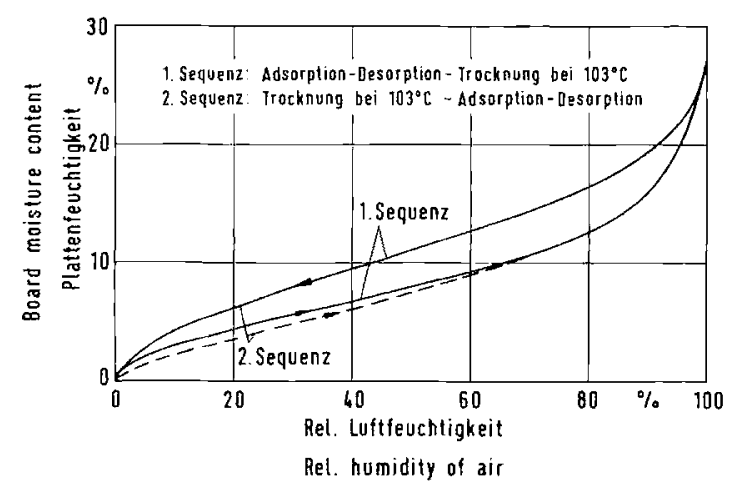

Resultate (Bild): 1. Die nach der ersten Sequenz ermittelten Adsorptionsisothermen lagen für alle drei Platten bis zu einer rel. Luftfeuchte von etwa $70 \%$ etwas höher als die nach der zweiten Sequenz bestimmten. 2. Im oberen Bereich der rel. Luftfeuchte nehmen die Adsorptionsisothermen nach den beiden Sequenzen den gleichen Verlauf. 3. Die Desorptionsisothermen, ermittelt nach beiden Sequenzen, sind ebenfalls deckungsgleich. 4. Die Ursache dieses Verhaltens dürfte in erster Linie am Vorhandensein von flüchtigen und zugleich stark hygroskopischen BestandteiIen in den Spanplatten liegen, die durch die Trocknung vor den Adsorptionsmessungen entfernt werden und somit die Hygroskopizität der Spanplatten etwas vermindern.

Meyer, B. 1983: Formaldehyde: Emission from particleboard postcured by radiofrequency heating. Holzforschung 37:41-45

\section{Holzeigenschaften von Notaphoebe ceratoxylon (Kosterm., spec. Inert.)}

Bandi Supraptono; H. Sachsse, Institut für Forstbenutzung der Universität Göttingen, Büsgenweg 4, D-3400 Göttingen

Subject: Investigation of wood properties of Notaphoebe ceratoxylon ("Medang tanduk") from Eastern Kalimantan.

Material und Methode: Die Medang-Arten Indonesiens (geschätzter Vorrat 148,8 Millionen $\mathrm{m}^{3}$ ) stellen ein großes, noch weitgehend ungenutztes holzwirtschaftliches Potential dar. Von der in Ostkalimantan besonders wichtigen Art Medang tanduk wurden an insgesamt 22 Stämmen von 2 verschiedenen Standorten technologisch wichtige, bisher unbekannte Holzeigenschaften untersucht.

Resultate: 1. Holzanatomie: Zerstreutporiges Laubholz mit vorwiegend einzeln stehenden, stark verthyllten Gefäßen. Zellartanteile: Gefäße 17,5; Faserzellen 64,5; Strahlparenchym 9,5; Längsparenchym 8,5\%. Kernvolumenanteil $64,3 \%$. 2. Darrdichte: $r_{0}=0,497 \ldots 0,514 \mathrm{~g} / \mathrm{cm}^{3}$. 3. Raumschwindma $3: \quad \beta_{V}=10,8 \% ; \quad$ Schwindungsanisotropie $1,76 \ldots 1,86$. 4. Stat. Festigkeiten und Elastizität: $\sigma_{\mathrm{dB}}=$ $40,3 \mathrm{~N} / \mathrm{mm}^{2} ; \sigma_{\mathrm{bB}}=82,3 \mathrm{~N} / \mathrm{mm}^{2} ; \sigma_{\mathrm{zB}}=112,3 \mathrm{~N} / \mathrm{mm}^{2} ; E_{\mathrm{b}}=$ $10450 \mathrm{~N} / \mathrm{mm}^{2}$. 5. Schlagzähigkeit: Versuchsfläche 1: $a=$ 36,1 ; Versuchsfläche 2: $a=46,9 \mathrm{~kJ} / \mathrm{m}^{2}$; signifikant unterschieden. 6. Holzfeuchte sehr hoch, langsame Trocknung. schlechte Tränkbarkeit. 7. Verleimung (Epoxyleim) erfüllt nicht die Bedingungen der DIN 68602. 8. Charakteristische Holzfehler: Markflecke, Fraßgänge holzbohrender Insektenlarven. 9. Holz für Zellstoff und Faserplatten geeignet.

In: Bandi Supraptono 1987: Untersuchung des technologischen Gebrauchswertes des Holzes von Medang tanduk (Kosterm., spec. Iner1.) aus der Familie der Lauraceen in Ostkalimantan. Diss. Forstwiss. FB der Univ. Göttingen 